\title{
Comparing Segmentation by Time and by Motion in Visual Search: An fMRI Investigation
}

\author{
Kevin Dent, Harriet Allen, and Glyn W. Humphreys
}

\begin{abstract}
Brain activity was recorded while participants engaged in a difficult visual search task for a target defined by the spatial configuration of its component elements. The search displays were segmented by time (a preview then a search display), by motion, or were unsegmented. A preparatory network showed activity to the preview display, in the time but not in the motion segmentation condition. A region of the precuneus showed (i) higher activation when dis-
\end{abstract}

\section{INTRODUCTION}

The human visual system is both flexible and limited in capacity. The visual environment is rich and presents more possibilities for visual analysis than the human brain can manage simultaneously (see Tsotsos, 1990, for a mathematical analysis). Mechanisms of selective attention have thus evolved to allow the flexible prioritization and deprioritization of different subsets of the visual input, according to moment-to-moment task demands.

Many studies have examined these flexible mechanisms of selection using the visual search task, in which an observer must find a target among varying numbers of distractors (see Wolfe, 1998, for a review). Search can be very difficult when the target is similar to a set of distractors (see Duncan \& Humphreys, 1989, 1992). Under these conditions of difficult search, performance is slow and shows strong effects of display size, as if each item must be selected and inspected individually using spatially selective mechanisms in order to determine target status (see Verghese, 2001; Treisman, 1988, for models of serial search).

However, the search process is opportunistic, taking advantage of simplifying strategies where possible. When relevant and irrelevant items are distinguished by a salient feature, search can be confined to items with the target feature, and items without this feature can be filtered out, substantially speeding performance. Differences in stereoscopic depth (Nakayama, \& Silverman, 1986), motion (McLeod, Driver, \& Crisp, 1988), and strong color differences (e.g., saturated red vs. green; Wolfe, Cave, \& Franzel, 1989) may all permit the selection of relevant groups. Additionally, in the preview paradigm, temporal differences in the presen-

The University of Birmingham plays were segmented by time or by motion, and (ii) correlated activity with larger segmentation benefits behaviorally, regardless of the cue. Additionally, the results revealed that success in temporal segmentation was correlated with reduced activation in early visual areas, including V1. The results depict partially overlapping brain networks for segmentation in search by time and motion, with both cue-independent and cue-specific mechanisms.

tation of the search display can be used to facilitate search. Specifically, if a set of irrelevant distractors is presented at least 400 msec before a later-appearing set, the earlier set may be effectively filtered out from search (e.g., Watson \& Humphreys, 1997).

The functional and neural mechanisms underlying efficient distractor rejection in each of these cases remain relatively poorly understood, although the temporal preview case has been perhaps most intensively studied (see Olivers, Humphreys, \& Braithwaite, 2006; Watson, Humphreys, \& Olivers, 2003). One important open question is whether segmentation and filtering by different cues relies on common, cue-independent (see Wolfe \& Horowitz, 2004 for one such proposal), or distinct cue-specific mechanisms (see McLeod et al., 1988; Nakayama \& Silverman, 1986, for stimulus-specific suggestions for the cases of motion and depth, respectively). The current article uses fMRI to separate out cue-invariant and cue-specific mechanisms contributing to segmentation by distinct transient cues in search, namely, a temporal preview and motion.

\section{Motion}

McLeod et al. (1988) were the first to examine the guidance of search by motion. In one illustrative experiment, search for an R in Qs and Ps was difficult when the displays were static, but was substantially easier if the R and Qs moved, consistent with observers selecting only the moving parts of the display for subsequent analysis. McLeod et al. (1988) initially put forward a bottom-up account of this result, built on stimulus-specific properties. They suggested that participants could use area MT of the brain as a "motion filter," to represent only the moving items, thus filtering-out 
the static items. Because MT cells represent coarse form information along with motion (e.g., Livingstone \& Hubel, 1987), these cells can signal the presence of a form singleton among the moving items. According to this "motion filter" account, segmentation is based on stimulus-specific features coded in specialized brain regions, and the mechanisms involved should overlap rather little with those involved in segmentation by other cues.

Ellison, Lane, and Schenk (2007) compared the effects of TMS on visual search for conjunctions of motion and orientation, as well as color and orientation. They showed that stimulation of parietal regions found to strongly disrupt color-orientation search failed to disrupt the motionorientation search condition, unless hMT/V5 was also stimulated at the same time. These results support the special status of conjunction search involving motion, and are consistent with the role of hMT/V5 as a motion filter.

Interestingly, not all motion cues give rise to efficient filtering in visual search. Cohen (1999) demonstrated that motion filtering required global displacement of the search items, even though cells in MT should respond to both local and global motion. Such results suggest that feature-specific filtering alone may not be sufficient to generate efficient search, and higher-level representations of surfaces, segmented by the visual cues, may be required to guide search (see Nakayama \& He, 1995). These higher-level representations may be cue-independent.

\section{Time (Temporal Preview)}

In addition to filtering based on the visual features of a set of items, a temporal difference between the onset of two sets of distractors ( $>400 \mathrm{msec}$ ) can be used to filter out early-appearing items, restricting search to later-appearing items (e.g., Watson \& Humphreys, 1997). Efficient filtering by temporal preview depends on participants attending to and then actively ignoring the initial distractors (e.g., Humphreys, Jung-Stalmann, \& Olivers, 2004; Humphreys, Watson, \& Jolicoeur, 2002; Watson \& Humphreys, 1997). Successful ignoring of the initial distractors is associated with activation of sites in the superior parietal lobe, the precuneus and cuneus, elicited in response to the preview display and preceding the search display (Allen \& Humphreys, 2008; Olivers, Smith, Matthews, \& Humphreys, 2005; Humphreys et al., 2004; Pollmann et al., 2003), even on "dummy" trials in which the initial distractors are not followed by search displays (Allen, Humphreys, \& Matthews, 2008; Pollmann et al., 2003).

Currently, it is unknown whether the activation of posterior parietal cortex, found when search items are segmented by a temporal preview, reflects preparation for a temporal signal (e.g., the onset of the second, search display) or cue-independent processes involved in segmentation into relevant and irrelevant groups. Interestingly, fMRI studies indicate that there can also be early activation when participants prepare to select motion-defined targets, prior to the stimuli appearing (Serences \& Boynton, 2007; Chawla,
Rees, \& Friston, 1999), but whether these preparatory states are specific to the type of processing subsequently undertaken or reflect more general mechanisms is unknown.

\section{The Current Study}

To assess cue-specific and cue-independent processes involved in segmentation and search, we directly compared the brain activity related to segmentation based on either motion or a temporal preview in a search task. Irrespective of the type of segmentation, the basic task required search for a target defined by a conjunction of form elements: an inverted $\mathrm{T}$ embedded in upright Ts and $90^{\circ}$ clockwise rotated Ts (Olivers, Watson \& Humphreys, 1999; Humphreys, Quinlan, \& Riddoch, 1989). This is a difficult search task in which there is no simple featural difference between the target and any of the distractors. However, prior evidence indicates that when the two sets of distractors are staggered over time, search can be confined to just the new items (Olivers et al., 1999). Additionally, other evidence (e.g., McLeod et al., 1988) indicates that motion may also be used to confine search to a moving set of letters in displays of heterogeneous moving and static letters. The current study manipulated the presence of motion and temporal preview segmentation cues to form three possible search displays. In the temporal preview segmentation condition, half of the items appeared for $2 \mathrm{sec}$ followed by the remaining items and the target participants should thus actively ignore the earlier appearing letters to gain an advantage in search. In the unsegmented and motion segmentation conditions, there were also two displays, matching the display sequence in the temporal (preview) segmentation condition. However, in the motion and unsegmented conditions, the initial items changed their locations when the second, search display appeared. Hence, there was minimal incentive for participants to attempt to actively ignore the initial items (although they contained a temporal cue to the search display, matching the temporal preview segmentation condition). In the motion segmentation condition in the second display, half the items including the target moved down the screen and half the items were static, thus participants should select only the moving items. In the unsegmented condition, all the items in the second display were static, and so there were no salient cues for segmentation. Following Allen et al. (2008) and Pollmann et al. (2003), a minority of "dummy" trials where the final search display did not appear were also included in order to isolate activity related to the first display alone.

\section{METHODS}

\section{Participants}

Sixteen participants were tested. Three were removed from analysis due to either poor behavioral performance or high head movement during data acquisition. 


\section{Design}

Three possible conditions involving different opportunities for segmentation were compared within participants: motion segmentation, temporal segmentation, and unsegmented. Additionally, trials were either standard trials with two displays first and second (75\%) or were dummy trials where only the first display was presented (25\%). Display size was 8 or 12 items.

\section{Stimuli}

The displays contained items constructed from two white lines of identical size, one horizontal and one vertical (each $0.7^{\circ} \times 0.1^{\circ}$, at a distance of $65 \mathrm{~cm}$ ), presented on a black background: inverted $\mathrm{T}$ (target), upright $\mathrm{Ts}$, and $90^{\circ}$ rotated Ts (distractors). Items were positioned in the cells of a regular $18 \times 16$ grid that subtended $15.3^{\circ} \times 12.7^{\circ}$ (minimum separation $0.1^{\circ}$; see Figure 1). No item was permitted to fall in either of the two central columns of cells. To avoid collisions when items were in motion, no more than one item appeared in each vertical column; the same constraint did not apply to horizontal rows. In all displays, the items were distributed evenly across the left and right sides of the screen.

In all conditions, the majority of trials consisted of two displays ( 2 sec duration). The first display was composed of ( 4 or 6 ) upright $\mathrm{T}$ distractors. The second display contained double the number of items ( 8 or 12), half the items were upright $\mathrm{T}$ distractors, and the remainder of the items were $90^{\circ}$ clockwise rotated $\mathrm{T}$ distractors, and the inverted $\mathrm{T}$ target (always present equally often on the left and the right).

In the temporal segmentation condition, the positions of the upright $\mathrm{T}$ distractors were held constant across the first and second displays, and participants should thus try to exclude the early-appearing distractors from search. In the motion segmentation and unsegmented conditions, there was no relation between the positions of the upright $T$ distractors across displays, and the first display was thus uninformative. In the unsegmented and temporal segmentation conditions, all items in the search display were static. In the motion condition in the second search display, the upright $\mathrm{T}$ items remained static whereas $90^{\circ}$ rotated $\mathrm{Ts}$ and the inverted $\mathrm{T}$ target moved down the screen at a constant speed of $3.2 \mathrm{deg} / \mathrm{s}$ (1 pixel every frame at $60 \mathrm{~Hz}$ ). Items were permitted to move through an additional two rows or $1.7^{\circ}$ below the bottom of the initial grid before scrolling off the bottom and reappearing gradually at the top of the grid. Participants could thus use motion as a cue to guide search.

In order to be able to measure preparatory brain activity to the first display only, "dummy" trials where only the first display appeared were included as a random $25 \%$ of trials in all blocks. On these trials, only the first display was presented and participants made no response and simply waited for the next trial. Because the timing of these trials was unpre-

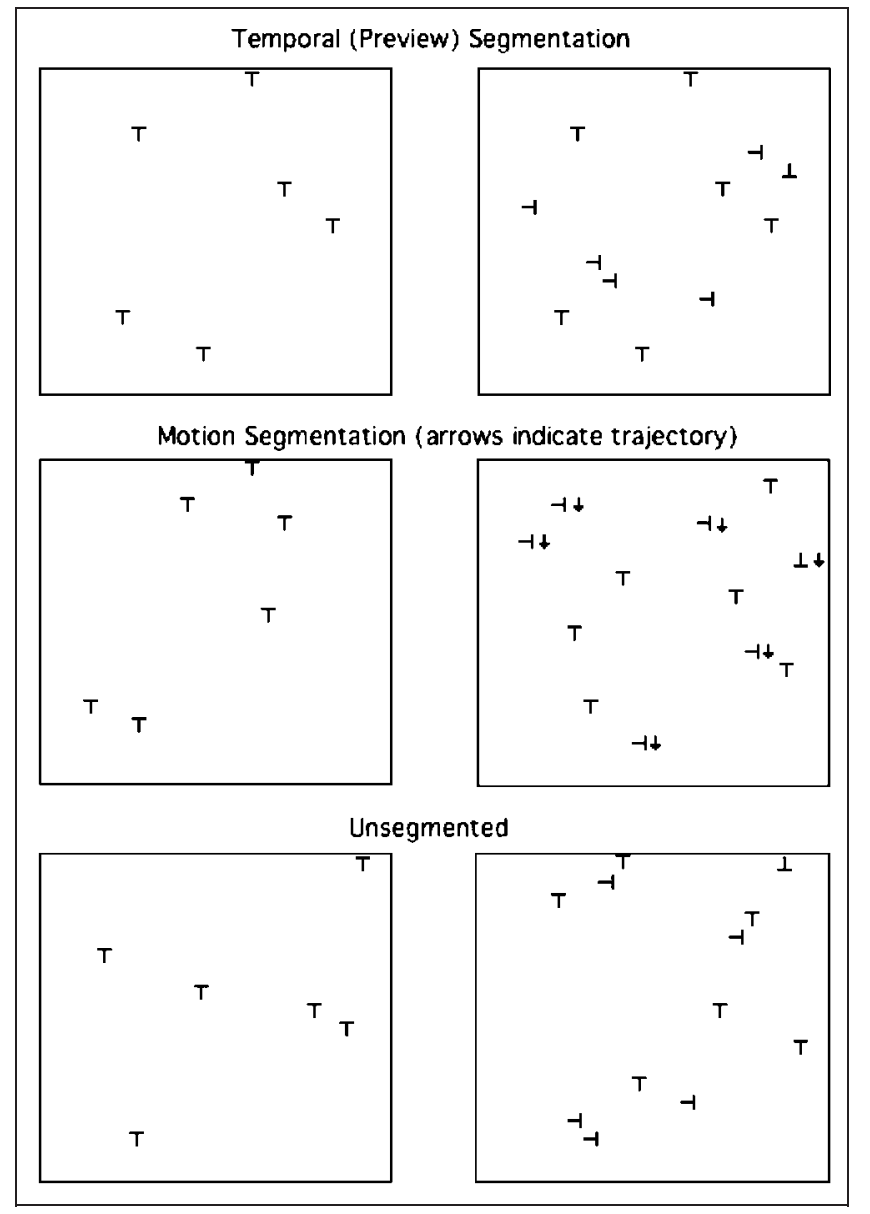

Figure 1. Stimulus examples with arrows indicating motion. In the experiment, the search items were white on a black background. The first display is shown on the left and the second display on the right. Note that the first display in all conditions consists of static, upright Ts.

dictable to the participant, only in the temporal segmentation condition should the initial display be treated as an informative preview.

\section{Procedure}

All participants took part in three functional scans (see Imaging Methods), each containing all conditions. Each condition was presented in a separate block of 16 trials, and each subject was presented with the conditions in a different order in each scan.

Participants were trained and practiced the task outside the scanner during the week prior to the scanning session. At the start of the experiment, participants were introduced to the scanner and reminded of the instructions. At the start of each trial block, a cue on the screen (3.5 sec duration) informed participants about the status of the first display. For blocks of trials in which the first display was irrelevant (i.e., motion segmentation and unsegmented conditions), the cue "DUMMY" appeared. For the temporal segmentation condition, the cue "PREVIEW" appeared. Each trial began with the presentation of a fixation cross for $0.5 \mathrm{sec}$. 
Trials within blocks were separated by a period of between 2 and $22 \mathrm{sec}$ (the duration was varied to optimize the imaging analysis). Three possible sequences of intertrial intervals were created, using optseq software (http://surfer.nmr. mgh.harvard.edu/optseq), and the sequences were assigned to blocks in a different order for each participant.

Participants responded to the search display by deciding if the target item appeared on the left or on the right by pressing one of two buttons held in the right hand. Set size was randomized in each block such that half of the trials contained 8 items and half contained 12 items. Any response occurring after the presentation duration of the search display (i.e., 2 sec) was recorded as incorrect.

\section{MRI Data Acquisition}

Imaging data were acquired using a Phillips 3-T Achieva scanner at Birmingham University Imaging Centre. A T1weighted $1 \times 1 \times 1 \mathrm{~mm}$ anatomical image was acquired for each participant. For the functional scans, three T2*weighted functional echo-planar imaging data were obtained using an eight-channel SENSE head coil with a sense factor of 2. Each scan consisted of 370 volumes ( $T R=2000 \mathrm{msec}$ ) of 34 slices, oriented just off axial, collected in ascending order (resolution $=3 \times 3 \times 3 \mathrm{~mm}$ voxels, $\mathrm{TE}=35 \mathrm{msec}$, flip angle $=65^{\circ}$, field of view $=240 \times 240 \times 102 \mathrm{~mm}$ ). At the beginning of each scan, at least two dummy scans were collected to allow stabilization of the magnetic field. Data from these scans were discarded.

\section{MRI Data Analysis Methods}

fMRI data processing was carried out using fMRI Expert Analysis Tool (FEAT) Version 5.91, part of FSL (FMRIB's Software Library; www.fmrib.ox.ac.uk/fsl).

For each participant's structural scan, nonbrain matter was removed using BET (Smith, 2002). This structural brain was then registered to the MNI template using FLIRT (Jenkinson, Bannister, Brady, \& Smith, 2002; Jenkinson \& Smith, 2001). For the functional scans, we applied motion correction using MCFLIRT (Jenkinson et al., 2002) and nonbrain matter was removed using BET (Smith, 2002). Prior to further analysis, the functional images were spatially smoothed with a Gaussian kernel of twice the voxel size (i.e., FWHM 6.0 mm). The entire 4-D data set from each scan was then grand-mean intensity normalized by a single multiplicative factor and a high-pass temporal filter applied (Gaussian-weighted least-squares straight line fitting, with sigma equal to $100.0 \mathrm{sec}$ ). Finally, the functional scans were registered to their participant specific anatomical scan and then the MNI standard brain using FLIRT (Jenkinson et al., 2001, 2002).

Each condition was modeled as a separate regressor, with the trials modeled as separate events. Contrasts between conditions were modeled within scan and then averaged separately across scans for each participant using a fixed effects model, by forcing the random effects var- iance to zero in FLAME (FMRIB's Local Analysis of Mixed Effects; Woolrich, Behrens, Bedell, Jenkinson, \& Smith, 2004; Beckman, Jenkinson, \& Smith, 2003). Group analysis data were then averaged using mixed effects analysis in FLAME stage 1 (Woolrich et al., 2004; Beckman et al., 2003). $Z$ (Gaussianized T/F) statistic images were thresholded using clusters determined by $Z>1.7$ and a (corrected) cluster significance threshold of $p=.05$ (Worsley, Evans, Marrett, \& Neelin, 1992).

\section{RESULTS}

\section{Behavioral Data}

Error rates were relatively high (between 3\% and 14\% across the conditions). It is likely that the strict response deadline imposed by the scanning procedure inflated errors in some conditions (e.g., unsegmented) more than others (responses after the deadline were recorded as errors). This is a problem encountered in similar fMRI search studies (Allen et al., 2008). For the behavioral analysis, we corrected response time by accuracy and analyzed efficiency, that is, response time/accuracy (see Townsend \& Ashby, 1983).

Incorrect RTs, and RTs more than 2 standard deviations from the mean of each cell of the design (4.2\%) were excluded, trials where no response was made within the 2-sec time window were treated as errors. Efficiency was then calculated as RT/proportion correct. A $3 \times 2$ within-participants ANOVA was conducted on the efficiency scores (Figure 2) with the factors of condition (preview, motion, neither) and display size $(8,12)$. There were reliable main effects of condition $[F(2,24)=25.543, p<.0001]$ and set size $[F(1$, $12)=22.304, p<.0001]$, but no interaction between the two $(F<1)$.

A series of planned contrasts (one-way repeated measures ANOVA) explored the overall difference between each pair of conditions. The presence of motion segmentation cues alone and the presence of an informative preview

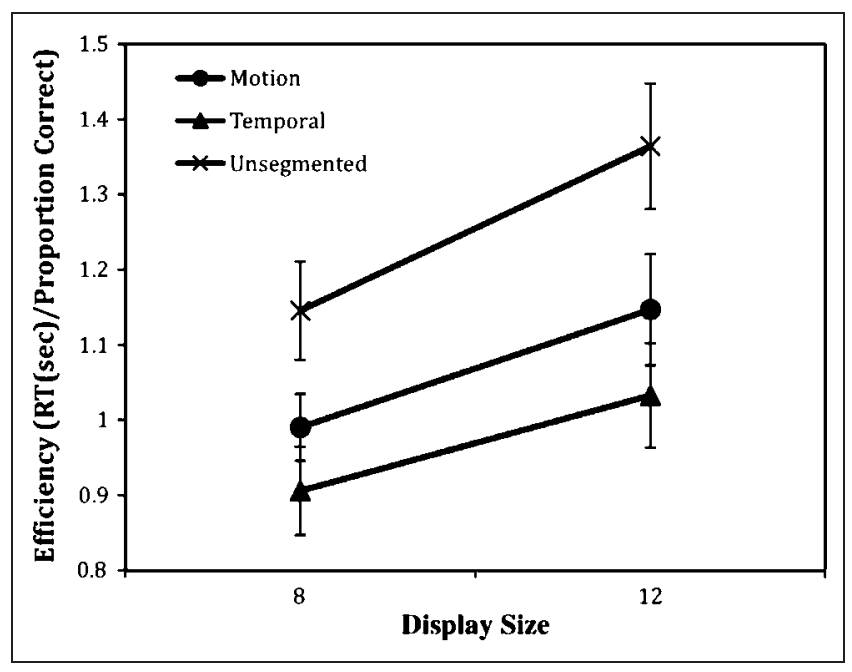

Figure 2. Efficiency [RT (sec)/proportion correct] as a function of display size ( $x$-axis) and condition (separate lines). 
Figure 3. BOLD activity for each contrast. (A) Preparatory activity in the temporal segmentation condition. Areas that were significantly more active in the temporal segmentation first display only condition than the motion segmentation first display only condition and the unsegmented first display only condition. Crosshairs at $-4,-84,24$. Left panel shows precuneus activation, central panel shows medial structures, and right panel illustrates both posterior precuneus and lateral temporo-occipital-parietal sites. (B) Activity during search. Activity during both the first and second displays. Red-yellow: Temporal segmentation activity greater than unsegmented search activity. Blue-light blue: Motion segmentation activity greater than unsegmented condition. Green: Overlap between red and blue. Crosshairs at $10,-56,30$.

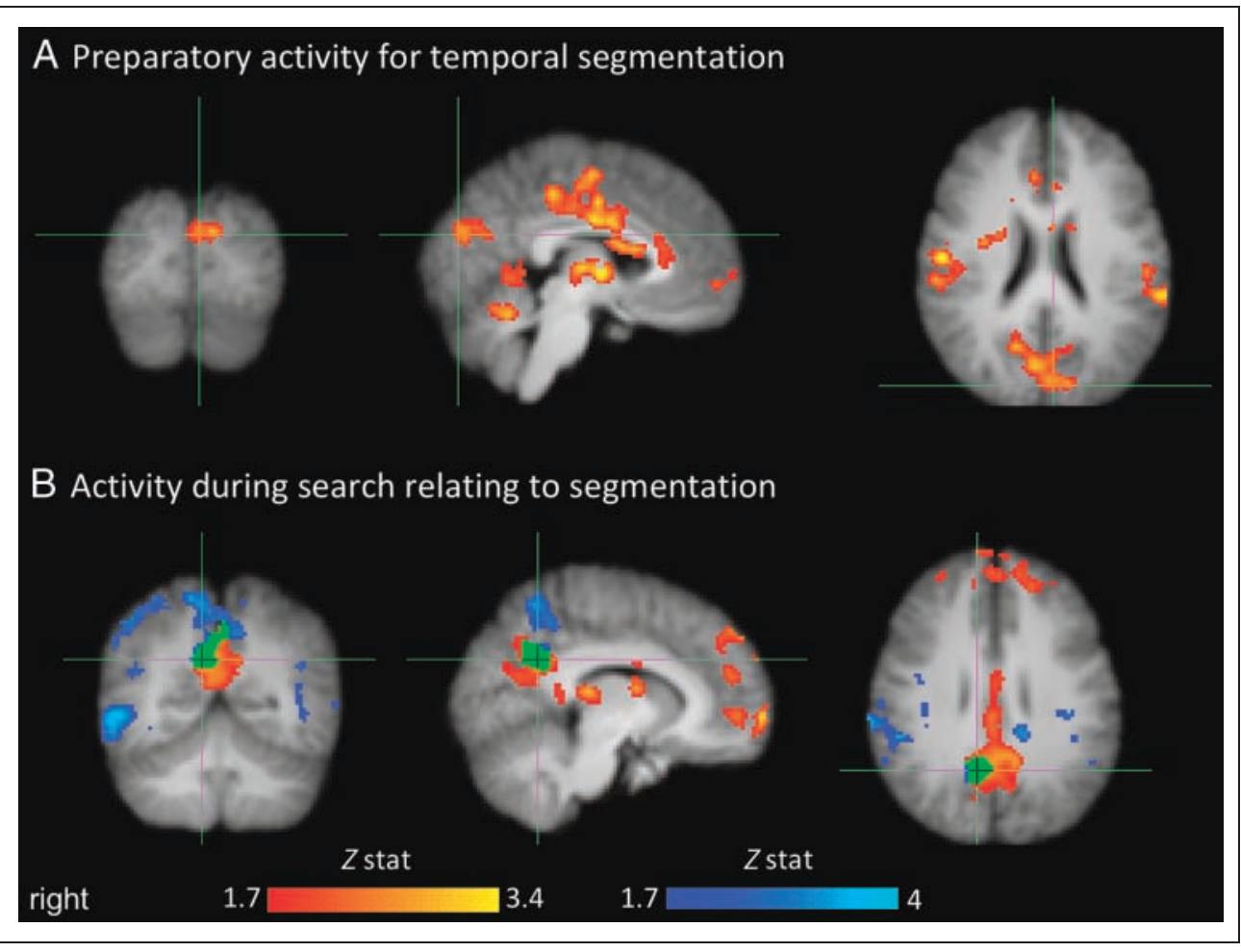

alone facilitated performance relative to when neither was present [motion: $F(1,12)=20.5, p<.001$; preview: $F(1$, $12)=44.6, p<.0001]$. The preview was more beneficial than the motion segmentation cue $[F(1,12)=11.1 p<.01]$.

\section{fMRI Data}

In our analysis and in contrast to some other previous studies of preview search (e.g., Allen et al., 2008), we were able to consider activation of the whole brain. The analytic strategy was as follows: We first considered preparatory activation by considering activation to the trials where only the first dummy display appeared, in each of the conditionstemporal segmentation, motion segmentation, and unsegmented. We then went on to consider activation in the nondummy two display trials, again for each of the segmentation conditions, compared to the unsegmented baseline. Finally, we consider correlations (positive and negative) between brain activation and behavioral performance.

\section{Preparatory Activation}

We isolated activation that was specifically related to the first displays (i.e., in the first display only "dummy" trials) and brain activations that were specific to each of the temporal and motion segmentation conditions (e.g., motion-temporal-unsegmented and temporal-motionunsegmented).

In the motion segmentation condition, there was no significant preparatory activity above that of the unsegmented baseline (also true in a simpler comparison of motion- unsegmented). In contrast, there was significant preparatory activity in a network of brain areas in the temporal segmentation condition (see Figure $3 \mathrm{~A}$ and Table 1).

In the temporal segmentation condition, the active preparatory areas consisted of a widespread network, including parts of the precuneus and cingulate cortex. Importantly, the site of activation found in the precuneus (illustrated in the left panel of Figure 3) is close to the critical preview activation found in this region in previous studies (e.g., Allen et al., 2008). Sites centered around the temporo-occipitalparietal junction were also active (lateral cluster in Table 1; right panel in Figure 3). There were some small foci of activation in frontal cortex, as well as activations in the cerebellum and in the thalamus (illustrated in Figure 3). In stark contrast, there was no preparatory activity at all in the motion segmentation condition. The data indicate that the preview search condition involved greater active preparation than the motion condition. Unlike in some prior studies (e.g., Chawla et al., 1999), there was no evidence for any significant top-down modulation or baseline shift behavior in the motion condition prior to the onset of the motion displays.

\section{Search Activation}

In a second set of analyses, we considered activity for the trials where two displays were presented, pooling activation for both the first and the second displays (using 4-sec trial regressors treating the onset of the first display as time 0 ). We isolated the brain areas that showed greater activation in either the motion segmentation or the temporal segmenta- 
tion conditions, compared to the unsegmented baseline. By comparing the two classes of segmentation, we identified areas common to both kinds of segmentation task (see Figure 3B and Table 2).

Temporal segmentation (preview). The analysis revealed a network of brain areas active during the temporal segmentation search task compared with the unsegmented baseline. Firstly, there was a set of medial brain areas including parts of the precuneus extending anteriorly into cingulate cortex. Secondly, there was a set of activations in frontal cortex corresponding to several distinct foci, superior and medial frontal gyri in both the left and the right, as well as a distinct focus in the right frontal pole. Thirdly, there was subcortical activation in the thalamus. Prior studies of search with temporally segmented displays have not used whole-brain analyses (e.g., Allen et al., 2008), and thus, they may have failed to detect the frontal activity observed here. The preparatory activity associated with temporal segmentation only overlapped to a small extent with the activity found here in the full search task. The primary focus of the preparatory activity measured in the precuneus in the "dummy" trials was adjacent but dorsal to the precuneus activity in the search task. Thus, from the current data, it appears that distinct areas are involved in (i) the preparation for ignoring old distractors and/or prioritizing new stimuli under preview conditions, and (ii) the guidance of search to targets on the basis of the initial segmentation.
Additionally, areas in the temporo-parietal junction (TPJ) that were active in the preparatory case were not active in the search display. Corbetta and Shulman (2002) have argued that the TPJ acts as a "circuit breaker," responding to the onset of new, behaviorally relevant stimuli. In the case of temporal segmentation, this region may be activated by the onset of the initial, behaviorally relevant distractors. It will not be activated by the occurrence of the initial distractors in the motion segmentation condition, however, as these items are behaviorally irrelevant. In search, any enhanced activation in the temporal segmentation condition may be balanced against greater activation through the more prolonged search in the unsegmented condition. The net result is no effect on the TPJ in search in the temporal segmentation condition. The TPJ does show activity in relation to successful preview search, when brain activity is probed with more fine-tuned regressors derived from computational models of search (Mavritsaki, Allen, \& Humphreys, 2010).

Also, in contrast to the preparatory activation (in the temporal segmentation condition), the search analysis revealed more widespread activation in the cingulate and more activity in more anterior frontal regions (in temporal segmentation compared with the unsegmented search condition). These frontal regions may be important in maintaining the search goal to ignore the old distractors (see Corbetta \& Shulman, 2002). In contrast to previous work (e.g., Olivers et al., 2005), we did not observe activation in the superior parietal lobe in the temporal segmentation search task

Table 1. Preparatory Activity

\begin{tabular}{|c|c|c|c|c|}
\hline Area & Peak & Subpeaks at & $Z_{\max }$ & Extent \\
\hline \multicolumn{5}{|l|}{ Medial Cluster (including Precuneus) } \\
\hline L. Fusiform gyrus (medial temporal) & $-34,-42,-20$ & extends to... & 3.3 & 12,242 \\
\hline L. Cerebellum & & $-10,-60,-22$ & 3.13 & \\
\hline L. Lingual gyrus (extends to precuneus) & & $-20,-44,-6$ & 3.12 & \\
\hline L Operculum (T-O-P junction) (temporal) & & $-58,-26,14$ & 3.1 & \\
\hline R. Cingulate [extends to both anterior (frontal) and posterior (parietal)] & & $12,22,14$ & 3.08 & \\
\hline L Operculum (T-O-P junction) & & $-58,-30,14$ & 3.08 & \\
\hline
\end{tabular}

\section{Lateral Cluster}
R. Superior temporal gyrus
R. Central operculum
R. Central operculum
R. Superior temporal sulcus
R. Postcentral gyrus
R. Superior temporal gyrus

$54,-2,-6$

2.88

2623

$\begin{array}{ll}54,-4,12 & 2.83 \\ 56,-2,2 & 2.81 \\ 54,-4,-2 & 2.81 \\ 56,-14,24 & 2.7 \\ 52,14,-12 & 2.68\end{array}$

Coordinates of activation during the first display of the temporal segmentation condition, that is, temporal segmentation first display only trialsmotion segmentation first display only trials-unsegmented first display only trials. Note that there were no significant areas activated in the equivalent contrasts for the motion first display trials, or unsegmented first display only trials. 
Table 2. Activity during Search

\begin{tabular}{|c|c|c|c|c|}
\hline Area & Peak Coordinates & Sub Peaks at & $Z_{\max }$ & Extent \\
\hline \multicolumn{5}{|l|}{ Temporal Segmentation-Unsegmented } \\
\hline \multicolumn{5}{|l|}{ Frontal cluster } \\
\hline L. Superior frontal gyrus & $-10,44,52$ & & 3.42 & 4307 \\
\hline R. Frontal pole & & $10,66,-2$ & 3.03 & \\
\hline R. Medial frontal gyrus & & $12,52,44$ & 2.91 & \\
\hline R. Superior frontal gyrus & & $22,50,42$ & 2.88 & \\
\hline L. Medial frontal gyrus & & $-28,22,40$ & 2.83 & \\
\hline L. Anterior paracingulate gyrus & & $-4,48,16$ & 2.83 & \\
\hline \multicolumn{5}{|c|}{ Medial cluster (including precuneus, cingulate, and thalamus) } \\
\hline R. Precuneus & $8,-56,32$ & & 3.08 & 3614 \\
\hline R. Precuneus & & $6,-64,40$ & 2.94 & \\
\hline R. Thalamus/Pulvinar & & $12,-28,12$ & 2.92 & \\
\hline L. Cingulate & & $0,-8,24$ & 2.85 & \\
\hline L. Posterior cingulate & & $-8,-48,28$ & 2.79 & \\
\hline L. Precuneus & & $0,-50,28$ & 2.78 & \\
\hline
\end{tabular}

\section{Motion Segmentation-Unsegmented}

Right cluster (including precuneus and cingulate)

R. Lateral occipital cortex (medial temporal gyrus)

R. Lateral occipital cortex (medial temporal gyrus)

R. Lateral occipital cortex (medial temporal gyrus)

R. Superior temporal gyrus

L. Cingulate (posterior)

R. Supramarginal gyrus

$$
\begin{array}{rr}
50,-70,8 & 3.93 \\
56,-62,0 & 3.46 \\
38,-72,8 & 3.36 \\
68,-36,8 & 3.36 \\
-14,-30,40 & 3.35 \\
60,-36,14 & 3.31
\end{array}
$$

Left lateral cluster

L. Lateral occipital cortex (medial temporal gyrus)

$$
-40,-78,0
$$

L. Lateral occipital cortex (medial temporal gyrus)

L. Lateral occipital cortex (medial temporal gyrus)

L. Parietal operculum T-O-P junction

L. Lateral occipital cortex (medial temporal gyrus)

L. Medial temporal gyrus

$$
\begin{array}{ll}
-46,-82,-6 & 3.59 \\
-56,-64,4 & 3.16 \\
-52,-30,16 & 2.98 \\
-52,-80,-2 & 2.96 \\
-60,-62,-2 & 2.81
\end{array}
$$

Coordinates of activation during both displays of each condition compared to both displays of the unsegmented condition.

(although this area did correlate positively with segmentation benefit, see below).

Motion segmentation. The motion condition also revealed activation in an extensive network of brain regions. Similarly to the temporal segmentation condition, this included activity in a set of medial brain structures including the cingulate and the precuneus. Importantly, we also observed activations in the lateral parietal lobe on the right including the supramarginal gyrus, and in areas of right temporal cortex, corresponding to motion processing areas (e.g., Huk, Dougherty, \& Heeger, 2002; Tootell et al., 1995).

Contrasting temporal and motion segmentation. A clear result emerged when we analyzed the overlap across the conditions (relative to the unsegmented baseline), with a large area of the precuneus being active for both 
temporal and motion segmentation. There were also areas involved in search involving temporal but not motion segmentation and in search involving motion but not temporal search. The frontal activations found for temporal segmentation (preview search) were not seen in search under motion segmentation conditions (confirmed by a direct subtraction of the two conditions, $p<.05$ ). Given the role of frontal structures in control processes (e.g., Corbetta \& Shulman, 2002), this finding supports the idea that the motion search involves a smaller contribution of executive control processes relative to temporal segmentation. There were also parietal activations and activations in temporal cortex that were present for motion but not temporal segmentation (preview search). The parietal activity here may reflect attentional tracking of moving items (Culham et al., 1998). The activations in posterior temporal cortex are likely related to the activations in low-level motion processing areas. (e.g., Huk et al., 2002; Tootell et al., 1995).

\section{Correlations with Behavior}

For each participant, the difference in efficiency (RT/proportion correct) between the cued condition (i.e., temporal, or motion segmentation) and the unsegmented baseline condition was taken as a measure of the benefit gained from the segmentation cue. We used these (demeaned) values as a new regressor in the fMRI data analysis to investigate whether there are areas that are more active when participants benefit more from preview (see Figure 4; statistics as in the fMRI Methods section).

Positive correlations. In the temporal segmentation condition, activation in the precuneus and cingulate, extending laterally to the superior parietal lobe on the right, correlated positively with the magnitude of the benefit (see Figure 4 and Table 3). The areas positively correlated with the motion segmentation benefit were more extensive. Similar to the temporal segmentation condition, there was a large area of the precuneus as well as areas within the superior parietal lobe (although in this case the activation was bilateral) extending into the inferior parietal lobe, that correlated positively with magnitude of benefit.

There were two major foci of overlap between the areas that correlated positively with segmentation benefit in each of the two segmentation conditions. Firstly, there was a large area of the precuneus that corresponded closely to the area of the precuneus overlapping in both tasks in the previous analysis of activation in search. There was also a second area of overlap in the right superior parietal lobe. This last result links to prior studies of preview search, where superior parietal activity has been associated with
Figure 4. Areas showing more BOLD activity in participants with greater benefits from segmentation (A) Activity during search that covaried positively with segmentation benefit. Activity during both the first and second displays. Red-yellow: Temporal segmentation activity greater than unsegmented search activity. Blue-light blue: Motion segmentation activity greater than unsegmented search activity. Green: Overlap between red and blue. Crosshairs at $-2,-56,32$. (B) Activity during search that covaried positively and negatively with preview segmentation benefit. Activity during both the first and second displays. Redyellow: Temporal segmentation activity with significant positive covariation with benefit, replotted. Blue-light blue: Temporal segmentation activity with significant negative covariation with benefit. In both cases, preview activity was compared to unsegmented condition activation. Crosshairs at $0,-52,18$. Images are in radiological format, that is, participant's right is shown on the left.

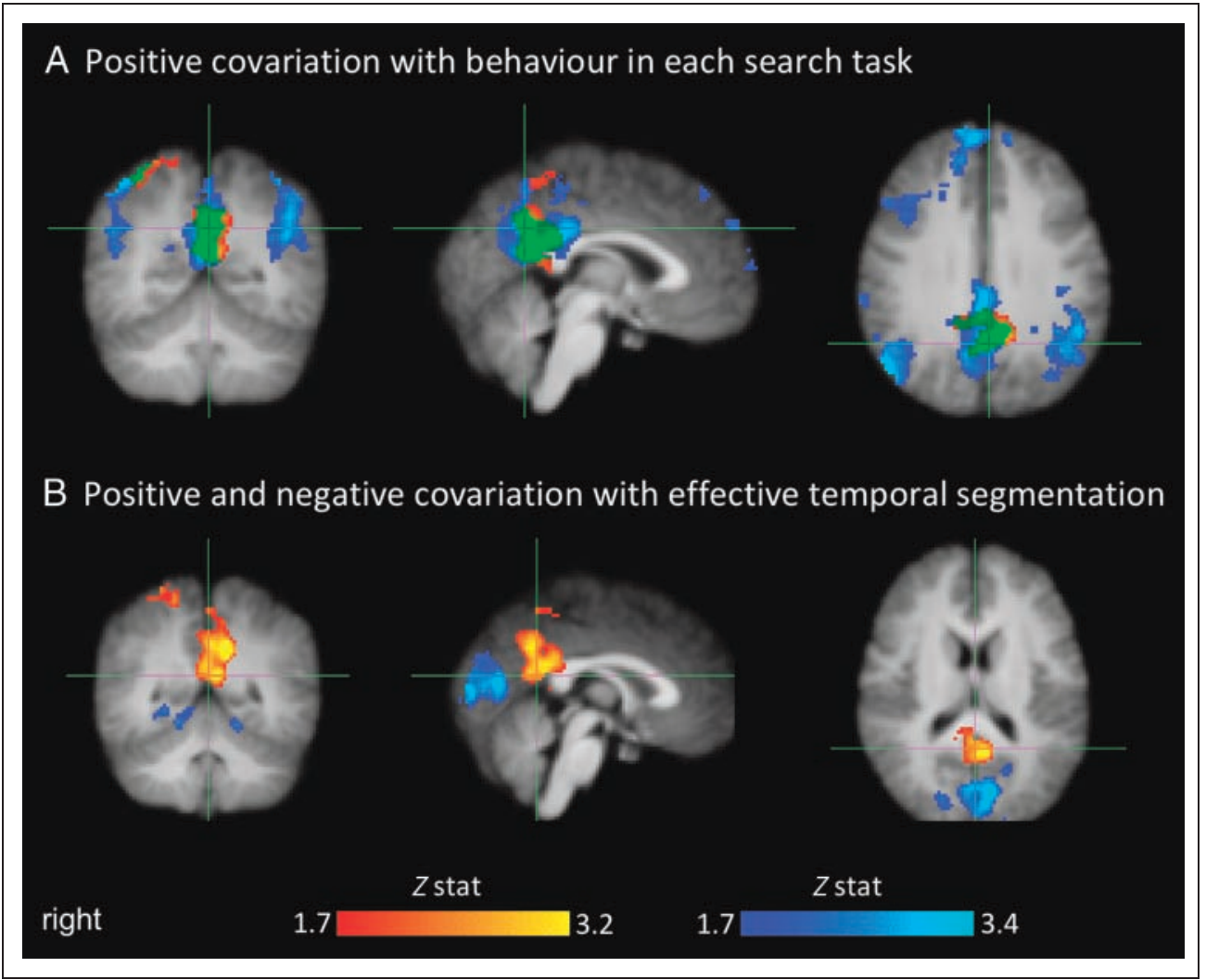


Table 3. Activity during Search That Showed Significant Positive Covariation with Performance over the Group

\begin{tabular}{|c|c|c|c|c|}
\hline Area & Peak Coordinates & Subpeaks at & $Z_{\max }$ & Extent \\
\hline \multicolumn{5}{|c|}{ Temporal Segmentation-Unsegmented } \\
\hline \multicolumn{5}{|c|}{ Parietal cluster (ext. to R. parietal) } \\
\hline R. Postcentral gyrus & $32,-32,72$ & & 3.21 & 2758 \\
\hline L. Cingulate (posterior) & & $-12,-52,32$ & 3.13 & \\
\hline R. Cingulate (posterior) & & $2,-46,24$ & 2.93 & \\
\hline L. Precuneus & & $-6,-54,18$ & 2.88 & \\
\hline L. Precuneus & & $-2,-54,34$ & 2.76 & \\
\hline R. Cingulate (posterior) & & $12,-30,52$ & 2.63 & \\
\hline
\end{tabular}

Motion Segmentation-Unsegmented

Medial cluster

R. cingulate (posterior)

$2,-38,28$

3.37

3313

L. Superior frontal gyrus

$-2,56,28 \quad 3.09$

R. Cingulate (posterior)

$2,-46,24 \quad 3.06$

R. Precuneus

$10,-66,24 \quad 3.06$

R. Precuneus

$0,-52,20$

3.01

R. Precuneus

$6,-64,38$

Frontal cluster

R. Superior frontal gyrus

$6,54,34$

3

2440
R. Superior frontal gyrus
R. Medial frontal gyrus
R. Superior frontal sulcus
R. Middle frontal gyrus
R. Superior frontal gyrus

$\begin{array}{cc}12,60,28 & 2.88 \\ 52,20,38 & 2.84 \\ 10,52,28 & 2.74 \\ 36,8,24 & 2.72 \\ 6,54,42 & 2.66\end{array}$

Left parietal cluster

L. Inferior parietal lobe

$-44,-64,52$

3.16

2394

L. Superior parietal lobule

$-24,-74,56 \quad 3.11$

L. Angular gyrus

$-46,-52,38$

3.1

L. Supramarginal gyrus

$-52,48,36$

3.05

L. Inferior parietal lobe

$-48,-64,46$

3.01

Right parietal cluster

R. Lateral occipital cortex (middle temporal gyrus)

$48,-60,22$

3.18

1783

R. Inferior parietal lobule

$52,-52,50 \quad 2.91$

R. Lateral occipital cortex (middle temporal gyrus)

$56,-64,28 \quad 2.89$

R. Superior parietal lobule

$42,-58,58 \quad 2.88$

R. Precentral sulcus

$30,-22,72$

2.86

Coordinates of activation during both displays of each condition compared to both displays of the unsegmented condition. 
contrasts between preview and nonpreview search conditions (e.g., Allen et al., 2008).

Negative correlations. In the temporal segmentation condition, activation of a largely symmetrical patch of occipital cortex, including parts of the calcarine sulcus and lingual gyrus, corresponding to area 17 or V1 and some extrastriate cortex (see Figure 4 and Table 4), correlated negatively with the behavioral benefit. No areas were negatively correlated with the behavioral benefit in the motion condition.

The outcome of this analysis is particularly striking. Larger benefits from temporal segmentation correspond to lower activation in early visual processing areas including primary striate cortex. This is not the case when search benefits from motion segmentation. This is the first evidence to tie the preview benefit specifically to lowered activation in early processing areas. The finding also dovetails nicely with psychophysical evidence that the preview benefit is associated with changes in sensory processing at the locations of distractors (e.g., Allen \& Humphreys, 2007a, 2007b). The results are consistent with distractors in preview search having attenuated processing (Watson \& Humphreys, 1997), and more so than distractors which are rejected under conditions of motion segmentation.

\section{DISCUSSION}

The current study compared the brain mechanisms underlying visual search where distractors could be segmented by time (under preview search conditions) and motion. Although search was substantially easier in the temporal and motion segmentation conditions, several brain areas showed raised levels of activation when the segmentation cues were present. In the temporal segmentation condi-

Table 4. Activity during Search That Showed Significant Negative Covariation with Performance over the Group

\begin{tabular}{|c|c|c|c|}
\hline Area & $\begin{array}{c}\text { Peak } \\
\text { Coordinates }\end{array}$ & $\begin{array}{c}\text { Subpeaks } \\
\text { at }\end{array}$ & $Z_{\max }$ Extent \\
\hline
\end{tabular}

Temporal Segmentation-Unsegmented

Visual cluster

\begin{tabular}{|c|c|c|}
\hline L. Calcarine & $-10,-82,8$ & 3.64 \\
\hline R. Calcarine & $6,-76,12$ & 3.28 \\
\hline R. Calcarine & $14,-82,6$ & 3.11 \\
\hline L. Supracalcarine & $-6,-86,20$ & 3.1 \\
\hline L. Lingual & $-10,-70,4$ & 3.03 \\
\hline R. Lingual & $22,-66,2$ & 2.75 \\
\hline
\end{tabular}

Coordinates of activation during both displays each condition compared to both displays of the unsegmented condition. Note that there were no areas significantly activated in the motion segmentation condition. tion (preview search), two sets of activation were separated. The first pattern of activation revealed a mostly parietal preparatory network where activation was raised when an informative preview occurred (compared with a noninformative baseline). The exact locus of the parietal activation found here was close but not identical to activation previously reported in preview search (Allen et al., 2008). These slightly different loci likely reflect small differences in the experimental and analytical approaches used (e.g., relatively larger face and house stimuli in Allen et al., 2008, compared with visually similar letters here). The preparatory activity in the preview condition is consistent either with participants inhibiting the initial distractors so they do not compete for selection with the new items, or with an expectancy being set for the properties of the new items (e.g., for new onsets).

In contrast to preview search, there was no evidence for greater preparatory activation in the motion condition compared with the unsegmented search baseline. This finding suggests that segmentation by motion does not involve preparation that is any different to the static search condition. Previous research has suggested that attention to motion is associated with increased activation or "baseline shifts" in motion processing areas (Serences \& Boynton, 2007; Saenz, Buracas, \& Boynton, 2002; Chawla et al., 1999). In the present case, however, the processes responsible for motion segmentation appear to operate in a fashion time-locked to stimulus onset rather than requiring active preparation.

In addition to these important differences in preparatory activity, analysis of activation associated with search performance revealed both common and distinct areas involved when temporal and motion segmentation occurred. In preview search, there was extensive frontal activation that was absent when motion segmentation occurred. This is consistent with the argument that there is greater top-down control when temporal rather than motion segmentation takes place (see Corbetta \& Shulman, 2002). In the motion but not in the preview case, there were also more rightlateralized superior parietal activations and activations in right-lateralized stimulus-specific motion processing areas in occipito-temporal cortex. We attribute these activations to (i) attentional tracking of the moving objects (e.g., Culham et al., 1998), and (ii) the recruitment of motion processing regions (e.g., Huk et al., 2002; Tootell et al., 1995)—processes demanded by the motion segmentation conditions.

As well as these distinct regions, several common areas were recruited in both tasks. Firstly, there was a region of the precuneus that was activated in both segmentation conditions compared with the unsegmented baseline. In addition to showing common activity in the temporal and motion segmentation conditions, this region also had an activity profile that was positively related to the magnitude of the behavioral benefit in each task (for temporal and for motion segmentation). Secondly, an area of right superior parietal cortex, primarily activated in the motion task, was also positively correlated with performance in both segmentation conditions. 
Concerning the activation found in the precuneus, the precuneus shows increased brain activation in a range of tasks (see Cavanna \& Trimble, 2006 for a review) including visuospatial imagery (e.g., Suchan et al., 2002). In their fMRI study of preview search, Allen et al. (2008) further demonstrated that a common area of the precuneus was activated both by preview search and by a spatial working memory task, with the preview benefit being disrupted by a spatial working memory load (see also Humphreys et al., 2002). One likely role played by the precuneus here may be to code a spatial representation of the distractor locations, allowing them to be filtered from search. Using behavioral data, Olivers et al. (1999) provided evidence that the preview benefit in the context of the current search task (e.g., conjunctions of form elements) was largely spatially based and dependent on a representation of the layout of the previewed items; a spatial "template." It is possible that the locations of the static distractors are similarly coded in motion search, and this is again mediated by the precuneus. An alternative view is that the precuneus codes the presence of separate surfaces formed by the segmented items, and codes the distinction between relevant and irrelevant groups, enabling one segmented set of items to be attended selectively (e.g., Nakayama \& He, 1995).

Now consider the region within superior parietal cortex that showed a positive correlation with performance in both temporal and motion segmentation tasks. Previous work has shown that this area is important in shifting attention both in space and between different visual features (see Serences, Liu, \& Yantis, 2005). Here superior parietal cortex may be involved when attention is switched into the relevant segmented group (the moving or the new items). This switching function may be additional to a role in multiple object tracking.

There were no brain regions that were negatively correlated with the motion search benefit. However, there was clear evidence for a negative correlation in the preview condition. In particular, the magnitude of the search benefit for the preview condition increased as there was reduced activity in early visual areas (including striate cortex). This deactivation may reflect the inhibition of distractor locations in preview search (see Humphreys et al., 2004; Watson \& Humphreys, 2000, for behavioral evidence from probe detection, and Allen \& Humphreys, 2007b, for evidence on contrast discrimination). An alternative proposal is that the apparent deactivation reflects the greater eye movements used in search compared with the preview condition. However, if eye movements were critical, then we would expect to see (negative) correlations between performance under preview conditions and activity in areas traditionally linked to eye movements, such as the frontal eye fields; there was no evidence for this. It is also difficult to see that a strategy of not making eye movements would only be employed under preview conditions. Any suppression of distractors was less evident in motion search. It may be that bottom-up segmentation in the motion case is sufficient for effective filtering, although we cannot rule out the possibility that early deactivation is present but somehow masked by the dynamic nature of the moving stimuli. Future research using other more sensitive techniques may reveal similar deactivation in the motion case. Recently, other authors have reported deactivation of visual cortex in response to unattended locations (e.g., Sylvester, Jack, Corbetta, \& Shulman, 2008; Serences, Yantis, Culberson, \& Awh, 2004). The present study is the first to link deactivation in early visual cortex to preview search.

In their study of preview search, Allen et al. (2008) considered a case of search where the previewed and search items were selected from different categories known to activate distinct functional regions of the brain (faces and houses). They found that previewing the distractors led to increased activation in stimulus-specific processing regions and this increase in activation was correlated with improved performance. These increases in activation contrast with the decrease in activation in V1 found in the current study. The functional significance in the difference in polarity of the stimulus-specific activations observed in the two studies is unknown, although it is possible that the active stimulus-specific regions (e.g., the fusiform face area) may "drive" inhibition in earlier brain regions. It also should be noted that this increase in activation was found during the first preview display as compared to the decrease in activation over the combined first and search displays. However, we should note that increases or decreases of activation may not be straightforwardly linked to behavioral psychophysical inhibition or facilitation. This remains a crucial topic for future research.

\section{Conclusions}

Our data suggest the following functional account of search when sets of distractors are segmented. To benefit from temporal but not from motion segmentation cues, individuals must prepare effectively to either ignore old items or to prioritize the selection of new stimuli. Successfully prepared individuals may be able to suppress old items, and this is associated with reduced activation in V1. In displays segmented by motion, however, the bottom-up signals may be sufficient to filter out irrelevant distractors, although these items still appear to activate early visual regions. Regardless of cue type, a region of the precuneus shows increased activation to the segmented compared to nonsegmented displays and correlates with behavioral performance. The precuneus activation likely corresponds to a spatial representation of the to-be-rejected distractors and/or a representation of segmented surfaces.

\section{Acknowledgments}

This work was supported by grants from the BBSRC and MRC to the third author and a grant from the ESRC to the second author.

Reprint requests should be sent to Kevin Dent, Behavioural Brain Sciences Centre, School of Psychology, The University of Birmingham, Edgbaston, Birmingham, B15 2TT, UK, or via e-mail:k.dent@bham.ac.uk. 


\section{REFERENCES}

Allen, H. A., \& Humphreys, G. W. (2007a). A psychophysical investigation into the preview benefit in visual search. Vision Research, 47, 735-745.

Allen, H. A., \& Humphreys, G. W. (2007b). Previewing distracters reduces their effective contrast. Vision Research, 47, 2992-3000.

Allen, H. A., Humphreys, G. W., \& Matthews, P. M. (2008). A neural marker of content-specific active ignoring. Journal of Experimental Psychology: Human Perception and Performance, 34, 286-297.

Beckman, C., Jenkinson, M., \& Smith, S. M. (2003). General multi-level linear modelling for group analysis in fMRI. Neuroimage, 20, 1052-1063.

Cavanna, A. E., \& Trimble, M. R. (2006). The precuneus a review of its functional anatomy and behavioural correlates. Brain, 129, 564-583.

Chawla, D., Rees, G., \& Friston, K. J. (1999). The physiological basis of attentional modulation in extrastriate visual cortex. Nature Neuroscience, 2, 671-676.

Cohen, D. J. (1999). Elements or objects: Testing the movement filter hypothesis. Journal of Experimental Psychology: Human Perception and Performance, 25, 348-360.

Corbetta, M., \& Shulman, G. L. (2002). Control of goal-directed and stimulus driven attention in the brain. Nature Reviews Neuroscience, 3, 201-215.

Duncan, J., \& Humphreys, G. W. (1989). Visual search and stimulus similarity. Psychological Review, 96, 433-458.

Duncan, J., \& Humphreys, G. W. (1992). Beyond the search surface: Visual search and attentional engagement. Journal of Experimental Psychology: Human Perception and Performance, 18, 578-588.

Ellison, A., Lane, A. R., \& Schenk, T. (2007). The interaction of brain regions during visual search processing as revealed by transcranial magnetic stimulation. Cerebral Cortex, 17, 2579-2584.

Huk, A. C., Dougherty, R. F., \& Heeger, D. J. (2002). Retinopy and functional subdivision of human MT and MST. Journal of Neuroscience, 22, 7195-7205.

Humphreys, G. W., Jung-Stalmann, B., \& Olivers, C. N. L. (2004). An analysis of the time course of visual marking using a probe-dot procedure. Perception \& Psychophysics, 66, 713-730.

Humphreys, G. W., Kyllingsbaek, S., Watson, D. G., Olivers, C. N. L., Law, I., \& Paulson, O. B. (2004). Parieto-occipital areas involved in efficient filtering in search: A time course analysis of visual marking using behavioural and functional imaging procedures. Quarterly Journal of Experimental Psychology, 57, 610-635.

Humphreys, G. W., Quinlan, P. T., \& Riddoch, M. J. (1989). Grouping processes in visual search, effects with single- and combined-feature targets. Journal of Experimental Psychology: General, 118, 258-279.

Humphreys, G. W., Watson, D. G., \& Jolicoeur, P. (2002). Fractionating the preview benefit in search: Dual-task decomposition of visual marking by timing and modality. Journal of Experimental Psychology: Human Perception and Performance, 28, 640-660.

Jenkinson, M., Bannister, P., Brady, M., \& Smith, S. (2002). Improved optimisation for the robust and accurate linear registration and motion correction of brain images. Neuroimage, 17, 825-841.

Jenkinson, M., \& Smith, S. M. (2001). A global optimisation method for robust affine registration of brain images. Medical Image Analysis, 5, 143-156.

Livingstone, M., \& Hubel, D. (1987). Psychophysical evidence for separate channels for the perception of form, color, movement, and depth. Journal of Neuroscience, 7, 3416-3468.

Mavritsaki, E., Allen, H. A., \& Humphreys, G. W. (2010) Decomposing the neural mechanisms of visual search through model-based analysis of fMRI: Top-down excitation, active ignoring and the use of saliency by the right TPJ. Neuroimage, 52, 934-946.

McLeod, P., Driver, J., \& Crisp, J. (1988). Visual search for a conjunction of movement and form is parallel. Nature, 332, 154-155.

Nakayama, K., \& He, Z. J. (1995). Attention to surfaces: Beyond a Cartesian understanding of visual attention. In T. V. Papathomas (Ed.), Early vision and beyond (pp. 69-77). Cambridge, MA: MIT Press.

Nakayama, K., \& Silverman, G. H. (1986). Serial and parallel processing of visual feature conjunctions. Nature, 320, 264-265.

Olivers, C. N. L., Humphreys, G. W., \& Braithwaite, J. J. (2006). The preview search task: Evidence for visual marking. Visual Cognition, 14, 716-735.

Olivers, C. N. L., Smith, S., Matthews, P., \& Humphreys, G. W. (2005). Prioritizing new over old: An fMRI study of the preview search task. Human Brain Mapping, 24, 69-78.

Olivers, C. N. L., Watson, D. G., \& Humphreys, G. W. (1999). Visual marking of locations and feature maps: Evidence from within-dimension defined conjunctions. Quarterly Journal of Experimental Psychology, 52, 679-715.

Pollmann, S., Weidner, R., Humphreys, G. W., Olivers, C. N. L., Muller, K., Lohmann, G., et al. (2003). Separating distractor rejection and target detection in posterior parietal cortex: An event related fMRI study of visual marking. Neuroimage, 18, 310-323.

Saenz, M., Buracas, G. T., \& Boynton, G. M. (2002). Global effects of feature-based attention in human visual cortex. Nature Neuroscience, 5, 631-632.

Serences, J., Liu, T., \& Yantis, S. (2005). Parietal mechanisms of switching and maintaining attention to locations, objects, and features. In L. Itti, G. Rees, \& J. Tsotsos (Eds.), Neurobiology of attention (pp. 35-41). New York: Academic Press.

Serences, J. T., \& Boynton, G. M. (2007). Feature-based attentional modulations in the absence of direct visual stimulation. Neuron, 55, 301-312.

Serences, J. T., Yantis, S., Culberson, A., \& Awh, E. (2004). Preparatory activity in visual cortex indexes distractor suppression during covert spatial orienting. Journal of Neurophysiology, 92, 3538-3545.

Smith, S. (2002). Fast robust automated brain extraction. Human Brain Mapping, 17, 143-155.

Suchan, B., Yaguez, L., Wunderlich, G., Canavan, A. G. M., Herzog, H., Tellmann, L., et al. (2002). Hemispheric dissociation of visuo-spatial processing and visual rotation. Cognitive Brain Research, 136, 533-544.

Sylvester, C. M., Jack, A. I., Corbetta, M., \& Shulman, G. L. (2008). Anticipatory suppression of nonattended locations in visual cortex marks target location and predicts perception. Journal of Neuroscience, 28, 6549-6556.

Tootell, R. B., Reppas, J. B., Kwong, K. K., Malach, R., Born, R. T., Brady, T. J., et al. (1995). Functional analysis of human MT and related visual cortical areas using magnetic resonance imaging. Journal of Neuroscience, 15, 3215-3230.

Townsend, J. T., \& Ashby, F. G. (1983). Stochastic modeling of elementary psychological processes. Cambridge, UK: Cambridge University Press.

Treisman, A. (1988). Features and objects: The fourteenth Bartlett memorial lecture. Quarterly Journal of Experimental Psychology, 40, 201-237. 
Tsotsos, J. K. (1990). A complexity level analysis of vision. Behavioral and Brain Sciences, 13, 423-455.

Watson, D. G., \& Humphreys, G. W. (1997). Visual marking: Prioritizing selection for new objects by top-down attentional inhibition of old objects. Psychological Review, 104, 90-122.

Watson, D. G., \& Humphreys, G. W. (2000). Visual marking: Evidence for inhibition using a probe dot detection paradigm. Perception \& Psychophysics, 62, 471-481.

Watson, D. G., Humphreys, G. W., \& Olivers, C. N. L. (2003). Visual marking: Using time in visual selection. Trends in Cognitive Sciences, 7, 180-186.

Wolfe, J. M. (1998). Visual search. In H. Pashler (Ed.), Attention (pp. 12-73). Hove, UK: Psychology Press.
Wolfe, J. M., Cave, K. R., \& Franzel, S. L. (1989). Guided search: An alternative to the feature integration model for visual search. Journal of Experimental Psychology: Human Perception and Performance, 15, 419-433.

Wolfe, J. M., \& Horowitz, T. S. (2004). What attributes guide the deployment of visual attention and how do they do it? Nature Reviews Neuroscience, 5, 1-7.

Woolrich, M. W., Behrens, T. E. J., Bedell, H. E., Jenkinson, M., \& Smith, S. M. (2004). Multi-level linear modelling for fMRI group analysis using Bayesian inference. Neuroimage, 21, $1732-1747$.

Worsley, K. J., Evans, A. C., Marrett, S., \& Neelin, P. (1992). A three-dimensional statistical analysis for $\mathrm{CBF}$ activation studies in human brain. Journal of Cerebral Blood Flow and Metabolism, 12, 900-918. 